Journal for ImmunoTherapy of Cancer

\title{
Immune checkpoint inhibition therapy for advanced skin cancer in patients with concomitant hematological malignancy: a retrospective multicenter DeCOG study of 84 patients
}

Ulrike Leiter, ${ }^{1}$ Carmen Loquai, ${ }^{2}$ Lydia Reinhardt, ${ }^{3}$ David Rafei-Shamsabadi (D) , ${ }^{4}$ Ralf Gutzmer, ${ }^{5}$ Katharina Kaehler, ${ }^{6}$ Lucie Heinzerling, ${ }^{7}$ Jessica C Hassel, ${ }^{8}$ Valerie Glutsch, ${ }^{9}$ Judith Sirokay, ${ }^{10}$ Nora Schlecht, ${ }^{11}$ Albert Rübben, ${ }^{12}$ Thilo Gambichler (1) ${ }^{13}$ Kerstin Schatton, ${ }^{14}$ Claudia Pfoehler, ${ }^{15}$ Cindy Franklin, ${ }^{16}$ Patrick Terheyden, ${ }^{17}$ Sebastian Haferkamp, ${ }^{18}$ Peter Mohr,${ }^{19}$ Lena Bischof, ${ }^{20}$ Elisabeth Livingstone, ${ }^{20}$ Lisa Zimmer, ${ }^{20}$ Michael Weichenthal (D) , Dirk Schadendorf, ${ }^{20}$ Andreas Meiwes, ${ }^{1}$ Ulrike Keim, ${ }^{1}$ Claus Garbe, ${ }^{1}$ Jürgen Christian Becker (D), ${ }^{21}$ Selma Ugurel (D) 20

To cite: Leiter U, Loquai C, Reinhardt L, et al. Immune checkpoint inhibition therapy for advanced skin cancer in patients with concomitant hematological malignancy: a retrospective multicenter DeCOG study of 84 patients. Journal for ImmunoTherapy of Cancer 2020;8:e000897. doi:10.1136/ jitc-2020-000897

- Additional material is published online only. To view please visit the journal online (http://dx.doi.org/10.1136/jitc2020-000897).

Accepted 27 September 2020

Check for updates

(C) Author(s) (or their employer(s)) 2020. Re-use permitted under CC BY-NC. No commercial re-use. See rights and permissions. Published by BMJ.

For numbered affiliations see end of article.

Correspondence to Professor Selma Ugurel; selma.ugurel@uk-essen.de

\section{ABSTRACT}

Background Skin cancers are known for their strong immunogenicity, which may contribute to a high treatment efficacy of immune checkpoint inhibition (ICI). However, a considerable proportion of patients with skin cancer is immuno-compromised by concomitant diseases. Due to their previous exclusion from clinical trials, the ICl treatment efficacy is poorly investigated in these patients. The present study analyzed the ICl treatment outcome in advanced patients with skin cancer with a concomitant hematological malignancy.

Methods This retrospective multicenter study included patients who were treated with ICl for locally advanced or metastatic melanoma (MM), cutaneous squamous cell carcinoma (CSCC), or Merkel cell carcinoma (MCC), and had a previous diagnosis of a hematological malignancy irrespective of disease activity or need of therapy at ICI treatment start. Comparator patient cohorts without concomitant hematological malignancy were extracted from the prospective multicenter skin cancer registry ADOREG. Treatment outcome was measured as best overall response, progression-free (PFS), and overall survival (OS).

Results 84 patients (MM, $n=52$; cSCC, $n=15$; MCC, $\mathrm{n}=17$ ) with concomitant hematological malignancy were identified at 20 skin cancer centers. The most frequent concomitant hematological malignancies were nonHodgkin's lymphoma $(n=70)$, with chronic lymphocytic leukemia $(n=32)$ being the largest entity. While 9 patients received $\mathrm{ICl}$ in an adjuvant setting, 75 patients were treated for advanced non-resectable disease (55 antiPD-1; 8 anti-PD-L1; 5 anti-CTLA-4; 7 combinations). In the latter 75 patients, best objective response (complete response+partial response) was $28.0 \%$, disease stabilization was $25.3 \%$, and $38.6 \%$ showed progressive disease (PD). Subdivided by skin cancer entity, best objective response was $31.1 \%$ (MM), $26.7 \%$ (CSCC), and $18.8 \%$ (MCC). Median PFS was 8.4 months (MM), 4.0 months (CSCC), and 5.7 months (MCC). 1-year OS rates were $78.4 \%$ (MM), $65.8 \%$ (cSCC), and $47.4 \%$ (MCC). Comparison with respective ADOREG patient cohorts without hematological malignancy $(n=392)$ revealed no relevant differences in ICI therapy outcome for $\mathrm{MM}$ and MCC, but a significantly reduced PFS for $\operatorname{CSCC}(p=0.002)$. Conclusions ICl therapy showed efficacy in advanced patients with skin cancer with a concomitant hematological malignancy. Compared with patients without hematological malignancy, the observed ICI therapy outcome was impaired in CSCC, but not in MM or MCC patients.

\section{BACKGROUND}

The introduction of immune checkpoint inhibitors (ICI) led to a tremendous change in the therapeutic management of advanced skin cancer. Previous to the ICI era, in Germany these standard therapies consisted mainly of different chemotherapies with or without combination with targeted agents. Patients with advanced malignant melanoma (MM) were treated with dacarbazine or temozolomide as single agents, or with combination chemotherapies like carboplatin plus paclitaxel or gemcitabine plus treosulfan; patients with advanced cutaneous squamous cell carcinoma (cSCC) received carboplatin, cisplatin, paclitaxel, or capecitabine as single agents or in combination with cetuximab; and patients with advanced Merkel cell carcinoma (MCC) were mainly treated with doxorubicin, 
paclitaxel, carboplatin, or etoposide as single agents or in combination. The objective response rates to standard therapies ranged between $10 \%$ and $20 \%$, with only short durations of response and limited patient survival. ${ }^{1-6}$ The ICI, particularly inhibitors of the PD-1/PD-L1 checkpoint (nivolumab, pembrolizumab, avelumab, cemiplimab), demonstrated strong treatment efficacies within clinical trials in MM, ${ }^{78} \mathrm{CSCC}^{9}{ }^{9}$ and MCC. ${ }^{10}{ }^{11}$ Approval of PD-1/ PD-L1 ICI was gained for nivolumab and pembrolizumab for MM, cemiplimab for cSCC, as well as avelumab and pembrolizumab for MCC. For MM, the CTLA-4 antibody ipilimumab is also approved as single agent and in combination with nivolumab. Response rates to first-line PD-1/PD-L1 ICI monotherapy amount to approximately $40 \%$ in MM, $50 \%$ in $\mathrm{CSCC}$, and $50 \%-70 \%$ in MCC. ${ }^{7-11}$ The high proportion of responders and the long duration of response observed in all three skin cancer entities might be based at least in part on their high immunogenicity caused by an UV-associated carcinogenesis which results in a high mutational burden and/or by a viral carcinogenesis. ${ }^{6}{ }^{12}$ However, these high treatment efficacies have only been demonstrated for immunocompetent patients, since the immuno-compromised patients were generally excluded from participation in clinical trials. The therapeutic efficacy of ICI in immunocompromised patients with skin cancer has therefore not yet been studied. In real-world populations, though, patients who are immuno-compromised by various causes such as concomitant malignancies, immunological disorders, or immuno-suppressive drug therapies represent a significant subgroup of patients with skin cancer. In addition, cSCC and MCC are known for their 10-fold increased incidence and their aggressive course of disease in immuno-compromised individuals. ${ }^{13}$ Our present study investigated real-world ICI treatment outcomes in advanced patients with skin cancer who are immuno-compromised by concomitant hematological malignancies.

\section{METHODS}

\section{Patient registry}

Patients presenting at academic cancer centers of the German Dermatologic Cooperative Oncology Group (DeCOG) between June 1, 2014 and May 31, 2019 were retrospectively identified according to the following inclusion criteria: histologically confirmed locally advanced or metastatic melanoma (MM), cSCC, or MCC, treated for their skin cancer disease with ICI (anti-PD-1; anti-PD-L1; anti-CTLA-4) as single agents or in combination; previous diagnosis of any hematological malignancy irrespective of its disease activity or need of therapy at start of ICI treatment for advanced skin cancer (this status was defined as "concomitant hematological malignancy"); detailed information on patient history including prior treatments; and follow-up information after start of ICI therapy. Data were extracted from local electronic patient files and captured within a central electronic data registry. Based on their relative frequency in this study, the hematological malignancies were assigned to three groups: chronic lymphocytic leukemia (CLL), other non-Hodgkin's lymphoma (NHL), and other (comprising all other entities not belonging to the previous two categories). Only the first ICI therapy received for advanced skin cancer was considered for this analysis. For all patient cohorts, treatment response was determined as best overall response recorded from the start of ICI treatment until disease progression or death, and was evaluated according to Response Evaluation Criteria in Solid Tumors (RECIST). ${ }^{14}$ During ICI therapy, the patients underwent regular staging procedures consisting of imaging techniques as CT, MRI or positron emission tomography-CT every 3 months. If there was suspicion of disease progression, staging was done earlier. Progression-free (PFS) and overall survival (OS) were defined as time from therapy start until disease progression or death, respectively; if no such event occurred, the date of the last patient contact was used as endpoint of survival assessment (censored observation). Median follow-up times were calculated from start of ICI therapy until last observation or death.

\section{Statistical analysis}

To check comparability between disease groups, clinical and demographic patient characteristics were evaluated. Numerical variables were described by median and IQR; patient cohorts were compared using Wilcoxon rank-sum test and two-sided $\chi^{2}$ tests, as appropriate. Survival probabilities with 95\% CIs were calculated using the KaplanMeier analysis. The two-sided log rank test was used for comparison of survival between groups. P values $<0.05$ were considered statistically significant. Statistical analyzes were performed with the statistical software SPSS V.21. Data analyzes for the patient cohorts with concomitant hematological malignancy were conducted from October 15, 2019 to December 15, 2019. Data analyzes for the comparator patient cohorts without concomitant hematological malignancy were done in August 2020.

\section{RESULTS \\ Patient characteristics}

A total of 84 patients with concomitant hematological malignancy were identified at 20 skin cancer centers according to the above described selection criteria; 52 patients with MM, 15 with CSCC, and 17 with MCC (table 1, figure 1). The median follow-up times were 8.7 months (IQR: 3.9-23.8) for MM, 6.2 months (IQR: 2.4-13.7) for CSCC, and 8.2 months (IQR: 4.0-13.2) for MCC. A total of nine patients received adjuvant treatment, of these eight patients suffered from MM, and one patient from MCC. For adjuvant ICI treatment, the median follow-up time was 3.4 months (IQR: 1.3-5.7) for MM patients, and 10.9 months for the MCC patient. For non-adjuvant ICI therapy, the median follow-up time was 13.5 months (IQR: 5.0-24.6) for MM, 6.2 months (IQR: 2.4-13.7) for cSCC, and 7.4 months (IQR: 3.8-14.2) 
Table 1 Patient characteristics

\begin{tabular}{|c|c|c|c|}
\hline & Hematological malignancy $\mathbf{N}(\%)$ & No hematological malignancy $\mathrm{N}(\%)$ & $P$ value \\
\hline MM (total) & $52(100 \%)$ & $257(100 \%)$ & \\
\hline Gender & & & 0.23 \\
\hline Male & $37(71.2 \%)$ & $157(61.1 \%)$ & \\
\hline Female & $15(28.8 \%)$ & $100(38.9 \%)$ & \\
\hline Age at diagnosis of skin cancer & & & n.s. \\
\hline Median (IQR) & 72.5 years $(59.0-76.0)$ & 67.0 years $(53.0-75.0)$ & \\
\hline Primary site of skin cancer & & & 0.38 \\
\hline Head and neck & $13(23.1 \%)$ & $51(19.8 \%)$ & \\
\hline Trunk & $21(40.4 \%)$ & $97(37.7 \%)$ & \\
\hline Extremities & $12(23.1 \%)$ & $48(18.6 \%)$ & \\
\hline Mucosa/others & $2(3.8 \%)$ & $27(10.4 \%)$ & \\
\hline Unknown primary & $4(7.6 \%)$ & $34(13.2 \%)$ & \\
\hline Median follow-up time (months, IQR) & $8.7(3.9-23.8)$ & $15.0(6.0-28.0)$ & n.s. \\
\hline cSCC (total) & $15(100 \%)$ & $59(100 \%)$ & \\
\hline \multicolumn{4}{|l|}{ Gender } \\
\hline Male & $12(80 \%)$ & $41(69 \%)$ & 0.65 \\
\hline Female & $3(20 \%)$ & $18(31 \%)$ & \\
\hline Age at diagnosis of skin cancer & & & n.s. \\
\hline median (IQR) & 76.0 years $(70.0-78.0)$ & $\begin{array}{l}77.0 \text { years } \\
(71.0-81.0)\end{array}$ & \\
\hline Primary site of skin cancer & & & 0.79 \\
\hline Head and neck & $13(86.7 \%)$ & $43(72.9 \%)$ & \\
\hline Trunk & $2(13.3 \%)$ & $9(15.3 \%)$ & \\
\hline Extremities & $0(0.0 \%)$ & $5(8,5 \%)$ & \\
\hline Mucosa & $0(0.0 \%)$ & $1(1.7 \%)$ & \\
\hline unknown primary & $0(0.0 \%)$ & $1(1.7 \%)$ & \\
\hline Median follow-up time (months, IQR) & $6.2(2.3-13.4)$ & $6.0(3.1-13.2)$ & n.s. \\
\hline MCC (total) & $17(100 \%)$ & $76(100 \%)$ & \\
\hline Gender & & & 0.82 \\
\hline Male & $12(70.6 \%)$ & $53(69.7 \%)$ & \\
\hline Female & $5(29.4 \%)$ & $23(30.3 \%)$ & \\
\hline Age at diagnosis of skin cancer & & & n.s. \\
\hline Median (IQR) & 70.0 years $(63.0-78.5)$ & 71.0 years $(62.7-78.0)$ & \\
\hline Primary site of skin cancer & & & 0.61 \\
\hline Head and neck & $5(29.4 \%)$ & $20(26.3 \%)$ & \\
\hline Trunk & $4(23.5 \%)$ & $13(17.1 \%)$ & \\
\hline Extremities & $7(41.2 \%)$ & $29(38.2 \%)$ & \\
\hline Unknown primary & $1(5.9 \%)$ & $14(18.4 \%)$ & \\
\hline Median follow-up time (months, IQR) & $8.2(4.0-13.2)$ & $11.4(4.1-19.1)$ & n.s. \\
\hline
\end{tabular}

Characteristics of all investigated patients treated with immune checkpoint inhibition (ICI) for advanced skin cancer. Comparisons are given with respective $p$ values for each skin cancer entity in $n=84$ patients with and $n=392$ patients without concomitant hematological malignancy. Percentages are given per column. Median follow-up times were calculated from start of ICI therapy until last observation or death. cSCC, cutaneous squamous cell carcinoma; MCC, Merkel cell carcinoma; MM, malignant melanoma.

for MCC. The comparator real-world cohorts without concomitant hematological malignancy comprised 257 patients with MM, 59 patients with cSCC, and 76 patients with MCC (table 1). The median follow-up times were 15.0 months (IQR: 6.0-28.0) for MM, 6.0 months (IQR:
3.1-13.2) for cSCC, and 11.4 months (IQR: 4.1-19.1) for MCC. No significant differences were detected within each skin cancer entity between patient cohorts with and without concomitant hematological malignancy (table 1). Within the patients with skin cancer with concomitant 


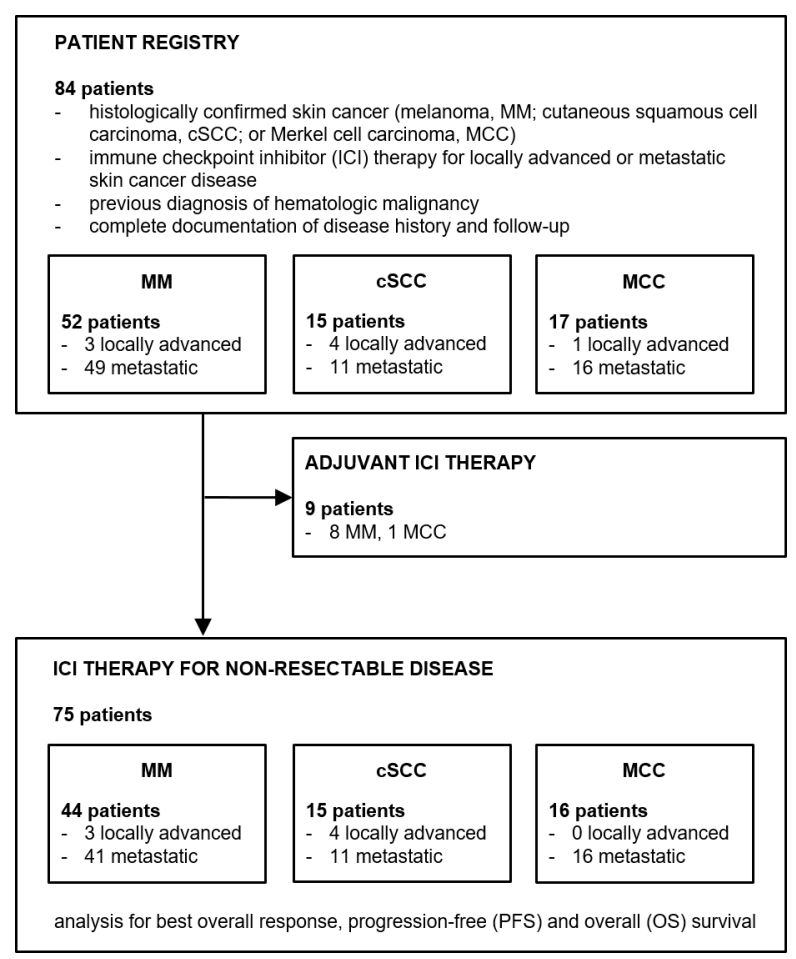

Figure 1 Schematic presentation of the registry and study flow for patients with skin cancer with concomitant hematological malignancy.

hematological malignancy, differences in the primary localization were observed between skin cancer entities as expected $(\mathrm{p}=0.006$; online supplemental table $\mathrm{S} 1)$ : most cSCC were localized on the head and neck (86.7\%), MM were predominantly localized on the trunk $(40.4 \%)$, and MCC were most often found on the extremities (41.2\%). No significant differences between skin cancer entities were found for gender $(\mathrm{p}=0.78)$, type of hematological malignancy $(\mathrm{p}=0.30)$, age at diagnosis of skin cancer $(\mathrm{p}=0.28)$, age at diagnosis of hematological malignancy $(\mathrm{p}=0.81)$, or type of treatment (non-resectable disease vs adjuvant; $\mathrm{p}=0.18$ ).

\section{Hematological malignancies}

The most common concomitant hematological malignancies in the total patient cohort $(n=84)$ were NHLs $(n=70$; $83.3 \%$ ), with CLL being the largest single entity ( $\mathrm{n}=32$; $38.1 \%$ ). Thus, for further analyzes we differentiated CLL from other NHL ( $\mathrm{n}=38 ; 45.2 \%)$ (table 2). Hematological malignancies other than NHL (Hodgkin's lymphoma, $\mathrm{n}=3$; polycythemia vera, $\mathrm{n}=3$; acute myeloid leukemia, $\mathrm{n}=2$; myelodysplastic syndrome, $\mathrm{n}=2$; chronic myeloid leukemia, CML, $\mathrm{n}=2$; systemic mastocytosis, $\mathrm{n}=1$; and myelofibrosis, $\mathrm{n}=1$ ) were less frequent and were therefore summarized in the category "other" $(\mathrm{n}=14 ; 16.7 \%)$. The frequencies of these three categories of hematological malignancies were balanced between the three skin cancer entities $(\mathrm{p}=0.30)$, while a higher rate of concomitant CLL was observed in cSCC (53.3\%) and MCC (47.1\%) as compared with MM (30.2\%) (table 2). Significant differences between the hematological disease categories were found in the disease-specific therapies: while $71.9 \%$ of CLL patients had not received any systemic treatment for their hematological malignancy prior to the start of ICI for their cutaneous malignancy, $57.9 \%$ of other NHL patients and $71.4 \%$ of patients with other hematological entities had received at least one therapy for their hematological malignancy ( $p=0.008$; online supplemental table S2). All patients were undergoing close clinical surveillance by their hemato-oncologists. Obvious differences were also present in the type of therapy for their hematological malignancy: unlike the other two disease categories, $55.7 \%$ of other NHL patients had received at least one chemotherapy before the start of ICI treatment, $44.7 \%$ had received rituximab, and $36.8 \%$ had received corticosteroids $(\mathrm{p}=0.07, \mathrm{p}=0.001$, and $\mathrm{p}=0.04$, respectively). No relevant differences were observed between hematological disease categories with regard to radiotherapy and stem cell transplantation $(\mathrm{p}=0.10, \mathrm{p}=0.35$; online supplemental table S2). At initiation of ICI therapy, only four patients $(4.8 \%$; $n=1$ with CLL; $n=1$ with other NHL; and $\mathrm{n}=2$ with other hematological malignancies) received

Table 2 Characteristics of patients with concomitant hematological malignancy

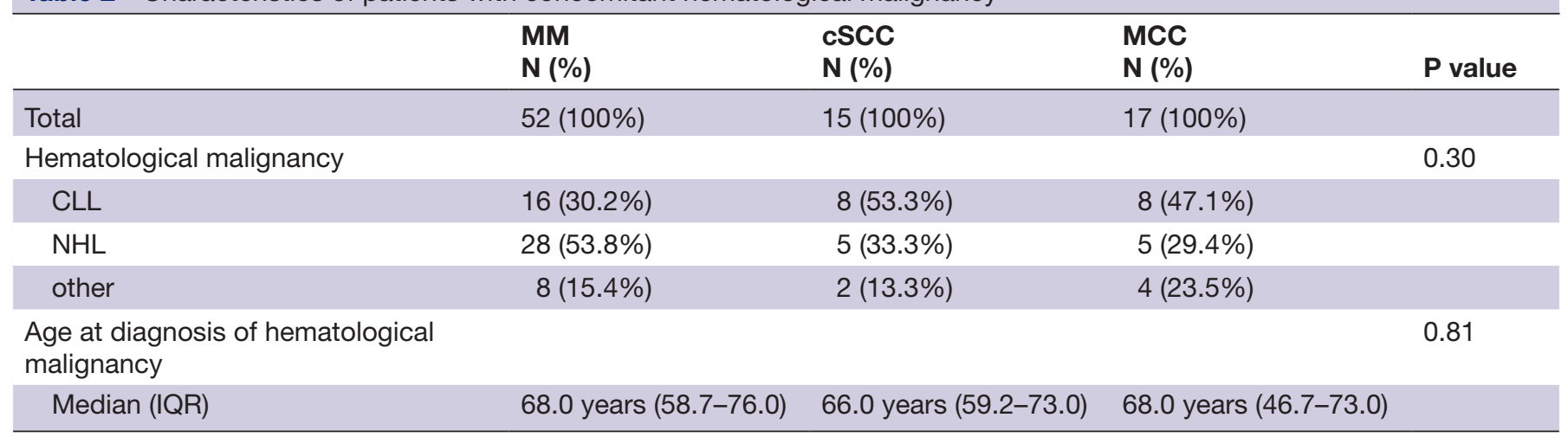

Characteristics of the total $n=84$ patient cohort. Percentages are given per column.

Hematological malignancies were categorized as chronic lymphocytic leukemia (CLL), other non-Hodgkin's lymphoma (NHL), and other (comprising all other entities not belonging to the previous two categories).

cSCC, cutaneous squamous cell carcinoma; MCC, Merkel cell carcinoma; MM, malignant melanoma. 
ongoing treatment for their hematological disease ( $p=0.19$; online supplemental table S2). All other patients had no indication for an active treatment of their hematological malignancy at start of ICI therapy. Notably, in the respective four patients, a progression of the hematological malignancy under ICI therapy was not reported.

\section{Patient characteristics at ICI treatment start}

At baseline of ICI therapy, the three skin cancer entities showed differences in terms of tumor load and stage of disease ( $\mathrm{p}=0.08$; online supplemental table S3). $26.7 \%$ of cSCC patients were treated for locally advanced disease, but only $5.8 \%$ of MM and $5.9 \%$ of MCC patients, respectively. $15.4 \%$ of MM, $5.9 \%$ of MCC, and none of cSCC patients received ICI therapy in the adjuvant setting. Regarding metastatic sites, $73.1 \%$ of MM patients showed visceral metastasis at ICI therapy start, whereas these were present in only $46.7 \%$ of cSCC and $52.9 \%$ of MCC patients ( $p=0.002$; online supplemental table S3). No relevant differences between the skin cancer entities were found regarding serum lactate dehydrogenase (LDH) levels $(p=0.28)$ and presence, type, and number of skin cancerspecific systemic pre-treatments $(\mathrm{p}=0.29, \mathrm{p}=0.39,0.32$; online supplemental table S3). cSCC and MCC patients received radiotherapy of their skin cancer disease more frequently than patients with MM $(53.3 \%, 47.1 \%$, and $17.0 \%$; $p=0.031$; online supplemental table S3). The stage of the concomitant hematological malignancy at start of ICI therapy for non-resectable skin cancer is provided in online supplemental table S4. Detailed characteristics of skin cancer disease at start of ICI therapy for both cohorts, patients with and without concomitant hematological malignancy, are provided in online supplemental table S5.

\section{Response to checkpoint inhibition therapy}

Of the total $n=84$ patients, $n=9$ received ICI therapy in an adjuvant setting, and $n=75$ for non-resectable skin cancer disease. Of the latter, $n=55$ received PD-1 ICI, $n=8$ PD-L1 ICI, $n=5$ CTLA- 4 ICI, and $n=7$ the combination of PD- 1 plus CTLA-4 ICI. While only MCC patients were treated with PD-L1 ICI, CTLA-4 ICI was received by MM patients only $(p<0.0001$; table 3$)$. Best overall response to ICI in the patient cohort treated for non-resectable disease was objective response (complete response (CR)+partial response (PR)) $28.0 \%$, disease stabilization (SD) $25.3 \%$, and progressive disease (PD) $38.6 \%$ (table 3). In six patients $(8.0 \%)$ a clear assessment of best overall response was not possible (not evaluable). The disease control rate $(\mathrm{CR}+\mathrm{PR}+\mathrm{SD})$ was $53.3 \%$. The tumor-specific objective response rates $(\mathrm{CR}+\mathrm{PR})$ were $31.8 \%$ for $\mathrm{MM}, 26.7 \%$ for cSCC, and $18.8 \%$ for MCC ( $\mathrm{p}=0.76$; table 3$)$. The best overall response to ICI therapy categorized according to the respective hematological malignancies did not show differences between the respective disease types and is presented in online supplemental table S6. On progression to ICI therapy, 31 patients underwent further treatment lines (table 3). Sixteen were MM patients of whom ten received another type of immunotherapy. For cSCC, seven patients received subsequent treatment, whereof five received chemotherapy. In the case of MCC, eight patients underwent further treatment, five of which received another type of immunotherapy.

\section{Survival under checkpoint inhibition therapy}

After a median follow-up time of 8.4 months, the median PFS after start of ICI therapy was 7.3 months (95\% CI 3.6 to 10.9$)$ for the total patient cohort $(\mathrm{n}=84)$; its 1-year PFS rate was $32.3 \%$ (95\% CI 20.6 to 44.6 ). Of the $n=75$ patients treated with ICI for non-resectable skin cancer, $25(33.3 \%)$ had died. Of those, $\mathrm{n}=13(29.5 \%)$ were $\mathrm{MM}$ patients, $n=5(33.3 \%)$ cSCC patients, and $n=7(43.8 \%)$ MCC patients; $\mathrm{p}=0.39$ (table 3 ). Cause of death was the skin cancer disease in most cases; only $12.0 \%$ of deaths occurred due to hematological malignancy with no relevant differences between the skin cancer entities $(\mathrm{p}=0.30$; table 3$)$. In patients treated with ICI for nonresectable disease, the median PFS was 6.3 months (95\%$\mathrm{CI}=2.5 ; 10.0$ ), the 1 -year PFS rate was $30.0 \%$ (95\% CI 18.7 to 41.3), the median OS was not reached, and the 1-year OS rate was $69.1 \%$ (95\% CI 574 to 80.8). Comparing the three skin cancer entities within the cohort of patients treated for non-resectable disease, the median PFS was 8.4 months (95\% CI 3.9 to 12.8 ) for MM, 5.7 months (95\% CI 0.7 to 10.7 ) for MCC, and only 4.0 months (95\% CI 0.3 to 7.9) for cSCC (table 3; figure 2A). Similarly, the 1 year PFS rate was $34.7 \%$ for MM, $35.3 \%$ for MCC, but only $11.8 \%$ for cSCC. 1-year OS rates were $78.4 \%$ (MM), $47.4 \%$ (MCC), and $65.8 \%$ (cSCC) (table 3; figure 2B). Neither relevant survival differences were found between patients grouped according to the type of their hematological malignancy (figure 2C,D), nor to the presence or absence of a previous systemic treatment of this hematological malignancy (data not shown). Abnormal blood counts, elevated or decreased from normal ranges, in patients with concomitant hematological malignancy (see online supplemental table S7) at baseline of ICI therapy did not correlate with survival outcomes in these patients (data not shown).

\section{Comparison with real-world patient cohorts without hematological malignancy}

Comparison of the ICI therapy outcome data of patients with concomitant hematological malignancy with realworld data of patient cohorts without hematological malignancy revealed no relevant differences for MM and MCC concerning PFS and OS on ICI treatment (see table 3, figure 3). For cSCC, the PFS was significantly reduced in patients with a concomitant hematological malignancy as compared with patients without (median 4.0 months vs not reached; $p=0.002$; table 3, figure 3). For OS no significant differences were found for either MM, MCC, and cSCC. Also, no significant differences were found for best overall response between the respective patients cohorts with and without concomitant hematological malignancy (table 3). 
Table 3 Characteristics and outcome of immune checkpoint inhibition therapy for non-resectable skin cancer

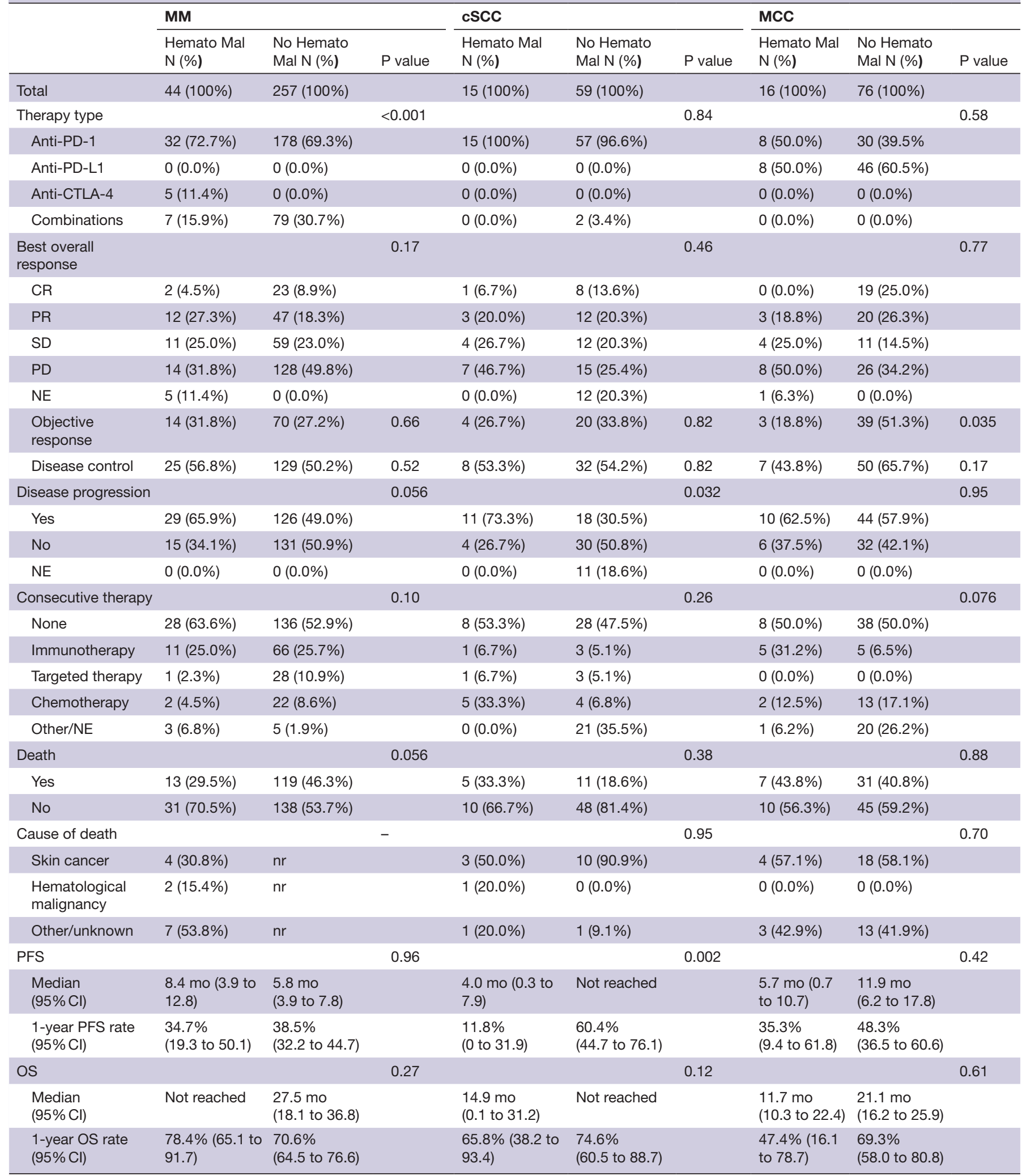

Data for immune checkpoint inhibition ( $\mathrm{ICl}$ ) therapy, outcome and follow-up are given for each skin cancer entity in $\mathrm{n}=75$ patients with and $\mathrm{n}=392$ patients without concomitant hematological malignancy (Hemato Mal). Treatment response is presented as best response recorded from the start of treatment until disease progression.

anti-CTLA-4, ipilimumab; anti-PD-1, nivolumab or pembrolizumab; anti-PD-L1, avelumab; CR, complete response; CR+PR, objective response; CR+PR+SD, disease control; cSCC, cutaneous squamous cell carcinoma; MCC, Merkel cell carcinoma; MM, malignant melanoma; mo, months; NE, not evaluable; nr, not reported; OS, overall survival; PD, progressive disease; PFS, progression-free survival; PR, partial response; SD, stable disease. 

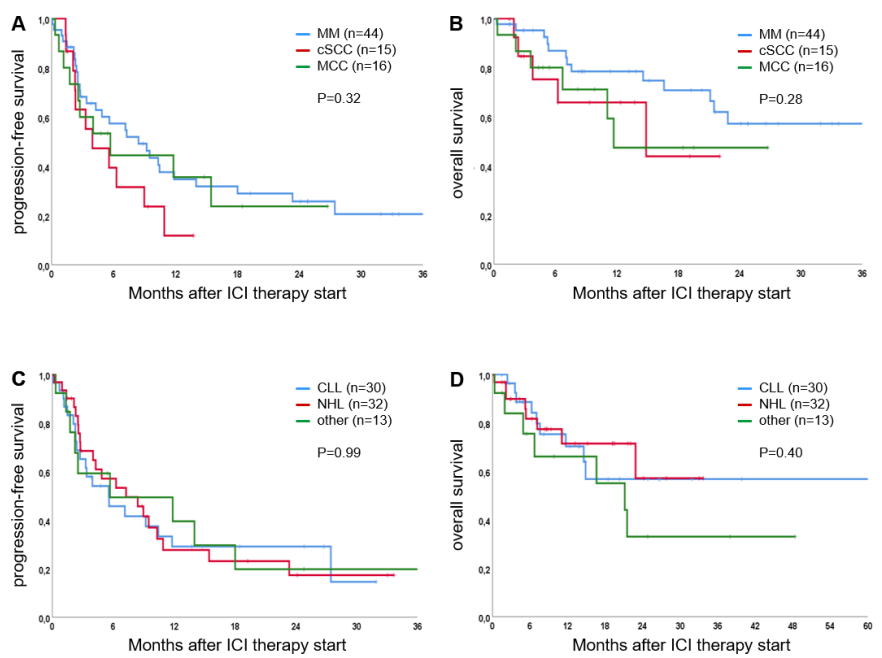

Figure 2 Survival analysis according to the skin cancer entities melanoma (MM), cutaneous squamous cell carcinoma (cSCC), and Merkel cell carcinoma (MCC) (A, B), and to the categories of hematological malignancy chronic lymphocytic leukemia (CLL), other non-Hodgkin's lymphoma (NHL), and other (comprising all other entities not belonging to the previous two categories) $(C, D)$, respectively, in $n=75$ patients treated with immune checkpoint inhibition (ICI) for non-resectable skin cancer. Kaplan-Meier curves showing the percentage of progression-free $(A, C)$ and overall $(B, D)$ survival after start of $\mathrm{ICI}$ treatment. $P$ values were calculated using the log rank test.

\section{DISCUSSION}

Patients with hematological malignancies are known for their susceptibility to develop skin cancers of high aggressiveness. These tumors are characterized by rapid growth and early metastasis, often showing only limited response to cytotoxic or targeted therapies. ${ }^{13}$ An epidemiological analysis in CLL and other NHL patients revealed an incidence of 1387 per 100,000 person-years for the development of cSCC in patients with other NHL, and of 2225 per 100,000 person-years in patients with CLL. ${ }^{15}$ In CLL patients, the cumulative recurrence rate of cSCC at 8 years after surgery was $13.4 \%$ as compared with $3 \%$ in immuno-competent patients. ${ }^{15}$ This high aggressiveness of cSCC in CLL patients is also reflected by their higher rate of metastatic disease and subsequent death from cSCC. ${ }^{16}$ Similar observations were reported for MM and MCC from studies analyzing the Surveillance, Epidemiology, and End Results database ${ }^{17}$ : in patients with a concomitant CLL, Brewer and coworkers found an impaired OS for both MM and MCC patients, as compared with the expected survival in patients without a hematological comorbidity. These findings were confirmed in a recent study of more than 150,000 patients with lymphoid neoplasms revealing an increased incidence and mortality for MM in patients with concomitant CLL and other NHL. ${ }^{18}$ However, it should be noted that this comparison is not equivalent across cancers, and studies on large patient cohorts are needed to gain detailed insights into these presumed causal relationships.

The high incidence and aggressiveness of skin cancers in patients with concomitant hematological malignancies has been mainly attributed to the impaired function of their
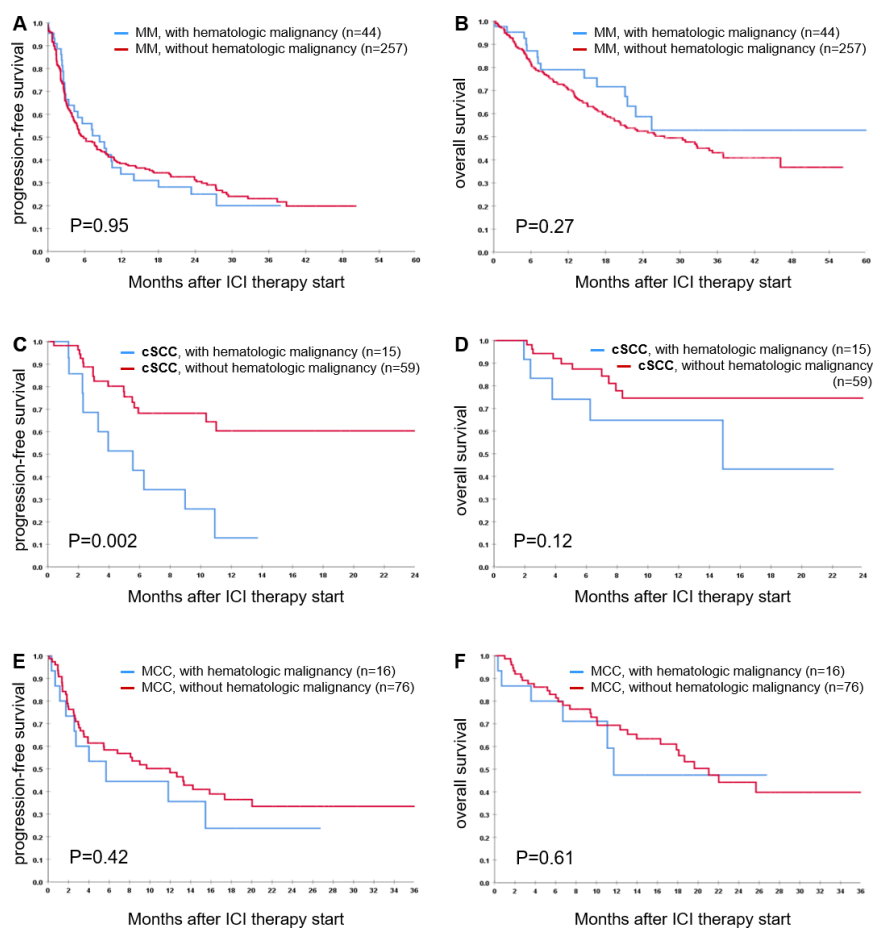

Figure 3 Survival analysis for patients with melanoma (MM) $(A, B)$, cutaneous squamous cell carcinoma (cSCC) $(C, D)$, and Merkel cell carcinoma (MCC) $(E, F)$ treated with immune checkpoint inhibition (ICl) for non-resectable disease. KaplanMeier curves showing the percentage of progression-free $(A$, $C, E)$ and overall $(B, D, F)$ survival after start of $\mathrm{ICI}$ treatment. Blue lines are survival curves of patients with concomitant hematological malignancy; red lines represent survival curves of the comparator patient cohorts without concomitant hematological malignancies derived from the real-world patient registry ADOREG. $P$ values were calculated using the log rank test.

immune system. ${ }^{13}$ This is particularly true for CLL patients, who are characterized by an impaired B cell function and functional defects of $\mathrm{T}$ cell subsets in terms of a decrease in helper $\mathrm{T}$ cell activity and an increase in regulatory $\mathrm{T}$ cell activity, leading to an elevated frequency of both secondary cancers as well as bacterial and viral infections. ${ }^{19-21}$ Furthermore, malignant CLL cells share phenotypic characteristics with regulatory B cells suggesting that they might negatively control T-cell activation and immune response. ${ }^{22}$ Also, the immuno-suppressive effects resulting from anticancer treatment for the hematological malignancy, particularly with chemotherapeutics or corticosteroids, might contribute to the patients' immuno-compromised state. This immunocompromised state of patients with hematological malignancies led to the assumption that immune-modulating therapies using ICI would result in only low or no treatment efficacy in these patients. This was one of the reasons why these patients were excluded from clinical trials investigating ICI therapies.

However, there is a high medical need to identify efficacious treatment options for advanced skin cancers in patients with concomitant hematological malignancies, as NHL including CLL is currently the 7th most frequent malignancy worldwide. $^{21}$ As data from clinical trials is missing, we collected 
and analyzed a cohort of 84 patients with concomitant hematological malignancies who received ICI for advanced skin cancer to gather information on treatment outcomes in daily clinical practice. To the best of our knowledge, this is the largest collection of such patients to date and was only made possible by the multicenter study design including 20 large academic skin cancer centers throughout Germany. This multicenter patient cohort contained 75 patients treated for non-resectable disease, who were evaluated in detail for ICI therapy response and survival. Best objective response $(\mathrm{CR}+\mathrm{PR})$ to ICI was low especially in cSCC $(26.7 \%)$ and MCC (18.8\%) patients as compared with data reported of clinical trials in comparable patient populations without concomitant hematological disease (50\% and $50 \%-70 \%$, respectively) ${ }^{9-11}$ Interestingly, this strongly impaired treatment response was not observed to the same extent in MM patients: herein, the MM patient cohort investigated by us showed a best objective response of $31.1 \%$ as compared with approximately $40 \%$ reported of fully immune-competent patients. ${ }^{78}$

As a next step, we intended to investigate the differences in ICI therapy outcomes of our real-world patient cohort with concomitant hematological malignacy in comparison not only to previously published highly selected clinical trial cohorts, but instead to real-world patient populations as comparators. Thus, we performed a comparison with the real-world data of respective MM, cSCC, and MCC patient populations derived from the prospective skin cancer registry ADOREG. For this analysis, we considered only those patients of the ADOREG registry, who were treated with ICI without concomitant hematological malignancy. Herein, we found a significantly impaired PFS ( $\mathrm{p}=0.002$ ) for cSCC patients, but no relevant difference in survival for MM and MCC patients (figure 3). Notably, the PFS and OS curves of MM and MCC patients with or without concomitant hematological malignancies were almost identical. It is apparent, that the realworld comparator patient cohorts derived from the ADOREG registry are characterized by a shorter PFS and OS compared with survival rates from landmark clinical trials. This can be explained by the well-known inferior treatment outcomes observed in real-world patient populations compared with selected populations investigated in randomized clinical trials. ${ }^{23}$ However, since our study patient population with concomitant hematological malignancy is a retrospectively collected real-world cohort, the comparison with the realworld ADOREG cohort appears to be more appropriate than the comparison with published clinical trial data.

The differential impact of concomitant hematological malignancies on ICI treatment outcome in MM and MCC versus cSCC patients may have several reasons. First of all, cSCC and also MCC appear to be under a more stringent immuno-surveillance than MM. Indeed, the relative increase in incidence in CSCC and MCC in immuno-compromised patients is much higher than for MM. ${ }^{26}{ }^{24}$ Thus, it can be assumed that once these tumors escaped the impaired immuno-surveillance caused by the hematological neoplasia, the beneficial effects of ICI therapy in the respective patients are less pronounced. Next, $15.9 \%$ of the MM patients investigated by us received an ICI combination therapy with ipilimumab plus nivolumab. This combination therapy was received by MM patients only, and not by CSCC or MCC patients. It is known to be more efficacious and to result in longer survival times than PD-1 monotherapy, ${ }^{25}$ so that the use of this combination in our MM patient cohort might be one major reason for their favorable therapy outcome. In patients with CLL, the use of the anti-CTLA-4 antibody ipilimumab may actually have an additional mode of action: it is well established that increased frequencies of CTLA-4 $4^{+}$$\mathrm{D} 4{ }^{+} \mathrm{CD} 25^{\text {high }}$ regulatory $\mathrm{T}$ cells are present in the peripheral blood of CLL patients ${ }^{26}$; furthermore, CTLA-4 expression not only mediates proliferation and survival of CLL cells, but also suppresses the costimulation of T cells. ${ }^{2728}$ Thus, ipilimumab may not only deplete regulatory $\mathrm{T}$ cells, but also the immuno-suppressive CTLA-4 ${ }^{+}$CLL cells. ${ }^{29}$ Another reason for the observed differences in therapy outcome may be the higher frequency of concomitant CLL disease in cSCC (53.3\%) compared with MCC (47.1\%) and MM (30.2\%). As discussed above, CLL is particularly known for its strong immuno-suppressive effect, even in stages in which no specific antileukemic therapy is indicated. ${ }^{19-21}$ Thus, in our investigated cohort CSCC and to a lesser extent MCC patients may be stronger immuno-compromised than MM patients.

With regard to effects of the ICI therapy on the patients' concomitant hematological malignancy, the four patients with active treatment of their hematological disease at start of ICI did not show a progression or worsening of their hematological disease under ICI therapy. Notably, all three patients who died from their hematological disease suffered from a concomitant CLL which was not requiring treatment at start of ICI. During ICI therapy these patients developed a rapid progression of their CLL with anemia (hemoglobin below $8.0 \mathrm{~g} / \mathrm{dL}$ ) as the major clinical sign. The other CLL patients treated with ICI revealed no relevant changes of their hematological disease state. Another 11 patients whose cause of death is unclear suffered from CLL $(n=4)$, NHL $(n=3)$, and other hematological malignancies $(n=4)$.

With respect to the limitations of our present study, its retrospective nature and the resulting potential patient selection bias have to be mentioned. Thus, it is of major importance to confirm our present results by prospective clinical trials or registries addressing patients with skin cancer with concomitant hematological malignancies. Moreover, our patient cohorts were highly heterogeneous with regard to the type and activity of their hematological disease. Almost all patients (95.2\%) did not receive treatment for their hematological disease at the start of ICI therapy for advanced skin cancer, and $51.2 \%$ had never received any disease-specific treatment. Accordingly, the hematological disease activity at start of ICI treatment did not reveal any relevant impact on therapy outcome, as well as peripheral blood cell counts for leukocytes, lymphocytes, and platelets did not (data not shown).

Taken together, our results indicate that patients with skin cancer with a concomitant hematological malignancy can benefit from ICI therapy. Compared with previously published data from immuno-competent patients, the therapeutic efficacy in this special patient subgroup is lower. 
However, using real-world patient cohorts as comparator, ICI therapy outcomes are comparable in patients with and without concomitant hematological malignancy in MM and MCC, but not in cSCC. In patients with CSCC, the PFS was significantly impaired in patients with concomitant hematological malignancy. These conclusions are of preliminary nature as they are resulting from retrospective cohorts, and therefore need confirmation by prospective trials or registries.

\section{Author affiliations}

${ }^{1}$ Department of Dermatology, University Hospital Tübingen, Tübingen, Germany ${ }^{2}$ Department of Dermatology, Johannes Gutenberg University Hospital Mainz, Mainz, Germany

${ }^{3}$ Skin Cancer Center at the National Center for Tumor Diseases Dresden, Department of Dermatology, University Hospital Carl Gustav Carus, Dresden, Germany

${ }^{4}$ Department of Dermatology and Venereology, University Medical Center Freiburg, Freiburg, Germany

${ }^{5}$ Skin Cancer Center, Department of Dermatology and Allergy, Hannover Medical School, Hannover, Germany

${ }^{6}$ Department of Dermatology, University Hospital Schleswig-Holstein - Campus Kiel, Kiel, Germany

${ }^{7}$ Department of Dermatology, University Hospital Erlangen, Erlangen, Germany

${ }^{8}$ Department of Dermatology and National Center for Tumor Diseases, University

Hospital Heidelberg, Heidelberg, Germany

${ }^{9}$ Department of Dermatology, University Hospital Würzburg, Würzburg, Germany

${ }^{10}$ Department of Dermatology, University Hospital Bonn, Bonn, Germany

${ }^{11}$ Department of Dermatology, Hospital Dortmund, Dortmund, Germany

${ }^{12}$ Department of Dermatology, University Hospital Aachen, Aachen, Germany

${ }^{13}$ Department of Dermatology, Ruhr University Bochum, Bochum, Germany

${ }^{14}$ Department of Dermatology, Heinrich Heine University Medical Faculty, Düsseldorf, Germany

${ }^{15}$ Department of Dermatology, Saarland University Medical Center, Homburg/Saar, Germany

${ }^{16}$ Department of Dermatology, University Hospital Cologne, Cologne, Germany

${ }^{17}$ Department of Dermatology, Universitätsklinikum Schleswig-Holstein - Campus Lübeck, Lübeck, Germany

${ }^{18}$ Department of Dermatology, University Hospital Regensburg, Regensburg,

Germany

${ }^{19}$ Department of Dermatology, Elbe Klinikum Buxtehude, Buxtehude, Germany

${ }^{20}$ Department of Dermatology, University Hospital Essen, University of Duisburg-

Essen, Essen, Germany

${ }^{21}$ Translational Skin Cancer Research, Deutsches Konsortium für Translationale Krebsforschung (DKTK), Essen, Germany

Contributors Study conception and design: UL, SU. Data acquisition: UL, CL, LR, DR-S, RG, KK, LH, JCH, VG, JS, NS, AR, TG, KS, CP, CF, PT, SH, PM, EL, LZ, MW, DS, AM, CG, SU. Data analysis and interpretation: UL, UK, JCB, SU. Writing of manuscript: UL, JCB, SU. Review and approval of manuscript: all authors.

Funding The authors have not declared a specific grant for this research from any funding agency in the public, commercial or not-for-profit sectors.

Competing interests UL: relevant financial activities (research support from Merck Sharp and Dohme; speakers and advisory board honoraria from Merck Sharp and Dohme, Novartis and Roche, Sanofi Aventis and travel support from Sun Pharma). CL: relevant financial activities (speakers, advisory board honoraria and travel support from Bristol Myers Squibb, Merck Sharp and Dohme, Merck Serono, Novartis, Roche, Pierre Fabre, Sun Pharma, Kiowa Kirin, Sanofi, Biontech, Allmiral Hermal). DR-S: relevant financial activities (speakers and advisory board honoraria from Novartis and Roche; travel support from Sanofy Genzyme, Roche and Novartis). JCH: relevant financial activities (research support from Bristol Myers Squibb; speakers and advisory board honoraria from Bristol Myers Squibb, Merck Sharp and Dohme, Novartis, Roche, Pierre Fabre and Sanofi Aventis, and travel support from Bristol Myers Squibb and Pierre Fabre). VG: relevant financial activities (honoraria from Bristol-Myers Squibb, advisory board honoraria from Novartis and reports travel support from Novartis, Pierre Fabre Pharmaceuticals, Bristol-Myers Squibb, Merck Sharp \& Dohme and Sanofi Genzyme). JS: relevant financial activities (speakers' honoraria and/or travel expense reimbursements from
Novartis, Bristol Myers Squibb, Merck Sharp and Dohme, Pierre Fabre and Roche). AR: relevant financial activities (travel grants and lecture fees from Bristol-Myers Squibb, Amgen and Roche). RG: relevant financial activities (personal fees and non-financial support from Bristol Myers Squibb, personal fees and non-financial support from Roche, grants, personal fees and non-financial support from Merck Serono, grants, personal fees and non-financial support from Amgen, personal fees and non-financial support from Pierre Fabre, personal fees and non-financial support from Sanofi Regeneron, personal fees from Merck Sharp and Dohme, grants, personal fees and non-financial support from Novartis, personal fees from Almirall Hermal, grants and personal fees from Pfizer, personal fees from SUN Pharma, personal fees from 4SC, grants from Johnson\&Johnson). KCK: relevant financial activities (research support, travel grants and honoraria from Bristol Myers Squibb and Merck Sharp and Dohme).TG: relevant financial activities (speakers and/or advisory board honoraria from Bristol Myers Squibb, Sanofi-Genzyme, Merck Sharp and Dohme, Novartis Pharma, Roche, Abbvie, Almirall, Janssen, Lilly, Pfizer, Pierre Fabre, Merck-Serono). KS: relevant financial activities (speakers and advisory board honoraria from AMGEN Oncology, Bristol Myers Squibb, Merck Sharp and Dohme, Novartis, Pierre Fabre and Roche, and travel support from Bristol Myers Squibb, Novartis, Pierre Fabre and TEVA/Cephalon Pharma). CP: relevant financial activities (speaker honoraria or honoraria as a consultant and travel support from Novartis, Bristol Myers Squibb, Roche, Merck Serono, Merck Sharp and Dohme, Celgene, AbbVie, Lilly and LEO). CF: relevant financial activities (travel support from Bristol Myers Squibb and Pierre Fabre; advisory board or honararia from Bristol Myers Squibb and Novartis). PT: relevant financial activities (speaker's honoraria from Bristol Myers Squibb, Novartis, Merck Sharp and Dohme, Pierre-Fabre, CureVac and Roche, consultant's honoraria from Bristol Myers Squibb, Novartis, Pierre-Fabre, Merck Serono, Sanofi und Roche and travel support from Bristol Myers Squibb, Pierre-Fabre and Roche). SH: relevant financial activities (research support from Bristol Myers Squibb; speakers and advisory board honoraria from Bristol Myers Squibb, Merck Sharp and Dohme, Novartis, Sanofi and Roche). PM: relevant financial activities (honoraria from Amgen, Bristol-Myers Squibb, Merck, Merck Sharp \& Dohme, Pierre Fabre, Novartis, Sanofi and Roche). LH: relevant financial activities (speakers and advisory board honoraria from Bristol Myers Squibb, Merck Sharp and Dohme, Merck Serono, Novartis, Amgen, Curevac, Pierre Fabre, Sanofi and Roche). EL: relevant financial activities (served as consultant or/ and has received honoraria from Amgen, Actelion, Roche, Bristol-Myers Squibb, Merck Sharp \& Dohme, Novartis, Janssen, Medac, and travel support from Amgen, Merck Sharp \& Dohme, Bristol-Myers Squibb, Amgen, Pierre Fabre, Sunpharma and Novartis). DS: relevant financial activities (Roche, Novartis, Bristol-Myers Squibb, Merck Sharp \& Dohme, Sanofi, Regeneron, Array, Pierre Fabre, 4SC, Helsinn, Philogen, InFlarX, Merck-Serono, SunPharma, Ultimovacs, Sandoz). CG: relevant financial activities (personal fees from Amgen, personal fees from Merck Sharp \& Dohme, grants and personal fees from Novartis, grants and personal fees from NeraCare, grants and personal fees from Bristol-Myers Squibb, personal fees from Pierre Fabre, personal fees from Philogen, grants and personal fees from Roche, grants and personal fees from Sanofi). JCB: relevant financial activities (speaker honoraria from Amgen, MerckSerono, Pfizer, Sanofi; advisory board honoraria from 4SC, Amgen, CureVac, eTheRNA, MerckSerono, Novartis and InProTher; research funding from Alcedis, Boehringer Ingelheim, Bristol-Myers Squibb, IQVIA, and MerckSerono; travel support from 4SC and Incyte). SU: relevant financial activities (research support from Bristol Myers Squibb and Merck Serono; speakers and advisory board honoraria from Bristol Myers Squibb, Merck Sharp and Dohme, Merck Serono, Novartis and Roche, and travel support from Bristol Myers Squibb, and Merck Sharp and Dohme). All other authors (Lena Bischof, Ulrike Keim, Andreas Meiwes, Lydia Reinhardt, Nora Schlecht) declared to have no conflicts of interest.

\section{Patient consent for publication Not required.}

Ethics approval The study was approved by the ethics committee of the University Duisburg-Essen (18-8404-B0). For all three skin cancer entities, comparator patient cohorts without concomitant hematologiclogical malignancy, but with otherwise identical inclusion criteria, were extracted from the prospective multicenter skin cancer registry ADOREG of the DeCOG. The ADOREG registry was approved by the ethics committee of the University Duisburg-Essen (14-5921-B0), and provides real-world data from skin cancer patients of clinical centers of the DeCOG.

Provenance and peer review Not commissioned; externally peer reviewed.

Data availability statement Data are available upon reasonable request. Original study data are available from the authors upon reasonable request.

Supplemental material This content has been supplied by the author(s). It has not been vetted by BMJ Publishing Group Limited (BMJ) and may not have been peer-reviewed. Any opinions or recommendations discussed are solely those of the author(s) and are not endorsed by BMJ. BMJ disclaims all liability and 
responsibility arising from any reliance placed on the content. Where the content includes any translated material, BMJ does not warrant the accuracy and reliability of the translations (including but not limited to local regulations, clinical guidelines, terminology, drug names and drug dosages), and is not responsible for any error and/or omissions arising from translation and adaptation or otherwise.

Open access This is an open access article distributed in accordance with the Creative Commons Attribution Non Commercial (CC BY-NC 4.0) license, which permits others to distribute, remix, adapt, build upon this work non-commercially, and license their derivative works on different terms, provided the original work is properly cited, appropriate credit is given, any changes made indicated, and the use is non-commercial. See http://creativecommons.org/licenses/by-nc/4.0/.

\section{ORCID iDs}

David Rafei-Shamsabadi http://orcid.org/0000-0002-7850-3199

Thilo Gambichler http://orcid.org/0000-0001-7862-3695

Michael Weichenthal http://orcid.org/0000-0002-9060-4961

Jürgen Christian Becker http://orcid.org/0000-0001-9183-653X

Selma Ugurel http://orcid.org/0000-0002-9384-6704

\section{REFERENCES}

1 Schadendorf D, Fisher DE, Garbe C, et al. Melanoma. Nat Rev Dis Primers 2015;1:15003.

2 Schadendorf D, van Akkooi ACJ, Berking C, et al. Melanoma. Lancet 2018;392:971-84.

3 Amaral T, Osewold M, Presser D, et al. Advanced cutaneous squamous cell carcinoma: real world data of patient profiles and treatment patterns. J Eur Acad Dermatol Venereol 2019;33 Suppl 8:44-51.

4 Hillen U, Leiter U, Haase S, et al. Advanced cutaneous squamous cell carcinoma: a retrospective analysis of patient profiles and treatment patterns-Results of a non-interventional study of the DeCOG. Eur J Cancer 2018;96:34-43.

5 Nghiem P, Kaufman HL, Bharmal M, et al. Systematic literature review of efficacy, safety and tolerability outcomes of chemotherapy regimens in patients with metastatic Merkel cell carcinoma. Future Oncol 2017;13:1263-79.

6 Becker JC, Stang A, DeCaprio JA, et al. Merkel cell carcinoma. Nat Rev Dis Primers 2017;3:17077.

7 Robert C, Long GV, Brady B, et al. Nivolumab in previously untreated melanoma without BRAF mutation. N Engl J Med 2015;372:320-30.

8 Ribas A, Puzanov I, Dummer R, et al. Pembrolizumab versus investigator-choice chemotherapy for ipilimumab-refractory melanoma (KEYNOTE-002): a randomised, controlled, phase 2 trial. Lancet Oncol 2015;16:908-18.

9 Migden MR, Rischin D, Schmults CD, et al. PD-1 Blockade with cemiplimab in advanced cutaneous squamous-cell carcinoma. $N$ Engl J Med 2018;379:341-51.

10 Kaufman HL, Russell J, Hamid O, et al. Avelumab in patients with chemotherapy-refractory metastatic Merkel cell carcinoma: a multicentre, single-group, open-label, phase 2 trial. Lancet Oncol 2016;17:1374-85.

11 Nghiem P, Bhatia S, Lipson EJ, et al. Durable tumor regression and overall survival in patients with advanced Merkel cell carcinoma receiving pembrolizumab as first-line therapy. J Clin Oncol 2019;37:693-702.

12 Yarchoan M, Hopkins A, Jaffee EM. Tumor mutational burden and response rate to PD-1 inhibition. N Engl J Med 2017;377:2500-1.

13 Collins L, Quinn A, Stasko T. Skin cancer and immunosuppression. Dermatol Clin 2019;37:83-94.

14 Therasse P, Arbuck SG, Eisenhauer EA, et al. New guidelines to evaluate the response to treatment in solid tumors. J Natl Cancer Inst 2000;92:205-16.

15 Brewer JD, Shanafelt TD, Khezri F, et al. Increased incidence and recurrence rates of nonmelanoma skin cancer in patients with nonHodgkin lymphoma: a Rochester epidemiology project populationbased study in Minnesota. J Am Acad Dermatol 2015;72:302-9.

16 Mehrany K, Weenig RH, Lee KK, et al. Increased metastasis and mortality from cutaneous squamous cell carcinoma in patients with chronic lymphocytic leukemia. J Am Acad Dermatol 2005;53:1067-71.

17 Brewer JD, Shanafelt TD, Otley CC, et al. Chronic lymphocytic leukemia is associated with decreased survival of patients with malignant melanoma and Merkel cell carcinoma in a SEER population-based study. J Clin Oncol 2012;30:843-9.

18 Herr MM, Schonfeld SJ, Dores GM, et al. Mutual risks of cutaneous melanoma and specific lymphoid neoplasms: second cancer occurrence and survival. J Natl Cancer Inst 2018;110:1248-58.

19 Christopoulos P, Pfeifer D, Bartholomé K, et al. Definition and characterization of the systemic T-cell dysregulation in untreated indolent B-cell lymphoma and very early CLL. Blood 2011;117:3836-46.

20 Forconi F, Moss P. Perturbation of the normal immune system in patients with CLL. Blood 2015;126:573-81.

21 Kipps TJ, Stevenson FK, Wu CJ, et al. Chronic lymphocytic leukaemia. Nat Rev Dis Primers 2017;3:16096.

22 Mohr A, Cumin M, Bagacean C, et al. The regulatory capacity of $\mathrm{B}$ cells directs the aggressiveness of CLL. Oncoimmunology 2019;8:1554968.

23 Khozin S, Blumenthal GM, Pazdur R. Real-World data for clinical evidence generation in oncology. J Natl Cancer Inst 2017;109. doi:10.1093/jnci/djx187

24 Plasmeijer El, Sachse MM, Gebhardt C, et al. Cutaneous squamous cell carcinoma (cSCC) and immunosurveillance - the impact of immunosuppression on frequency of cSCC. J Eur Acad Dermatol Venereol 2019;33 Suppl 8:33-7.

25 Larkin J, Chiarion-Sileni V, Gonzalez R, et al. Five-Year survival with combined nivolumab and ipilimumab in advanced melanoma. $N$ Engl J Med 2019;381:1535-46.

26 Beyer M, Kochanek M, Darabi K, et al. Reduced frequencies and suppressive function of CD4+CD25hi regulatory $T$ cells in patients with chronic lymphocytic leukemia after therapy with fludarabine. Blood 2005;106:2018-25.

27 Mittal AK, Chaturvedi NK, Rohlfsen RA, et al. Role of CTLA4 in the proliferation and survival of chronic lymphocytic leukemia. PLoS One 2013;8:e70352.

28 Do P, Beckwith KA, Cheney C, et al. Leukemic B cell CTLA-4 suppresses costimulation of T cells. J Immunol 2019;202:2806-16.

29 Arce Vargas F, Furness AJS, Litchfield K, et al. Fc effector function contributes to the activity of human anti-CTLA-4 antibodies. Cancer Cell 2018;33:649-63. 